\title{
Poder y educación superior: perspectivas de jóvenes universitarios
}

Power and higher education: narratives of young university students Poder e educaçãouniversitária: perspectivas de jovens universitários

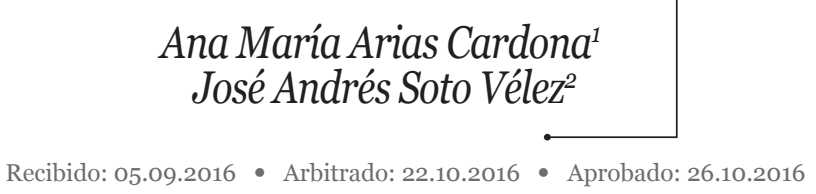

\section{Resumen}

El poder es una realidad inherente a toda relación humana que se configura en la complejidad de diversas dinámicas. En este artículo de la tesis doctoral sobre relaciones sociales ${ }^{3}$ en la universidad, se exponen los resultados de dos subcategorías que hacen parte de la categoría de Poder: el poder concebido como una asimetría inevitable; y las prácticas de resistencia como intentos de revertir lo instituido. En el trabajo de campo se realizaron 14 talleres con metodología participativa y 23 entrevistas a profundidad que dieran razón de su diseño cua-

${ }^{1}$ Psicóloga y especialista en Psicología Clínica, énfasis: Salud mental de la Universidad Pontificia Bolivariana de Medellín. Magíster en Educación y Desarrollo Humano y Doctora en Ciencias Sociales: Niñez y Juventud del Centro de estudios avanzados en niñez y juventud conformado por la Fundación Centro Internacional de Educación y Desarrollo Humano - CINDE y la Universidad de Manizales. Docente tiempo parcial Corporación Universitaria Lasallista. Email: anamaria2468@gmail.com / anarias@lasallistadocentes.edu.co

${ }^{2}$ Auxiliar de Investigación en GIPA (Grupo de Investigación en Psicología Aplicada) de la Corporación Universitaria Lasallista. Estudiante de Psicología en la Corporación Universitaria Lasallista - Caldas (Antioquia). Email: joseandres0823@hotmail.com

${ }^{3}$ Relaciones sociales es una categoría muy amplia, configurada por muchos elementos que interactúan entre sí; pero para el caso de la tesis doctoral se trabajaron tres categorías: poder, conflicto y pluralidad. Este artículo se centra en la presentación de algunos resultados sobre la categoría poder. 
litativo y enfoque hermenéutico. Participaron 119 jóvenes registrados en programas de las Facultades de Ciencias Sociales y Educación de universidades públicas y privadas (4 campus y 6 sedes), ubicadas en el departamento de Antioquia (Colombia).Se identificó que los universitarios son conscientes de la presencia del poder en las relaciones con sus pares e incluso con la institución misma, mantienen una visión negativa del poder, le asocian con el abuso y el autoritarismo, suelen ubicarlo en un actor externo y muy pocas veces se reconocen como quienes lo detentan y como caso atípico emergen algunas prácticas de resistencia en una de las universidades públicas, en donde se asocia el poder con el uso de la violencia como la única manera, hasta el momento, que ellos encuentran para revertir lo instituido.

Palabras clave: Educación superior, poder, perspectivas y jóvenes universitarios

\section{Abstract}

The power is a reality inherent in every human relationship, and is configured in the complexity of different dynamics. In this article of the doctoral thesis on social relations in the University, presented the results of two subcategories that make part of the category of Power, Known: the power conceived as an asymmetry inevitable; and the practices of resistance as attempts to reverse the instituted. In the work of field is used some techniques of data collection (14 workshops with participatory methodology and 23 in-depth interviews) to give reason for their qualitative design and a hermeneutic approach. Participated 119 young registered in programs of the faculties of Social Sciences and Education of public and private universities (4 campuses and 6 sites), located in the department of Antioquia (Colombia). Finally, it was identified that the university students are aware of the presence of power in relations with their peers and even with the institution itself, maintain a negative view of power, you associated with abuse and authoritarianism, Often placed in an external actor and very few times are recognized as those who possess it and as atypical case emerge certain practices of resistance in one of the public universities, where the 
power is associated with the use of violence as the only way, until the moment that they find to reverse what had been instituted.

Keywords: Higher education, power, perspectives and university students.

\section{Resumo}

O poder é umarealidadeinerente a toda relação humana que se configura na complexidade de diversas dinâmicas. Neste artigo da tese doutoral sobre relaçõessociaisna universidade, se expõem os resultados de duassubcategorias que fazem parte da categoria de Poder: o poder concebido como umaassimetriainevitável; e as práticas de resistência como tentativas de reverter o instituído. No trabalho de campo se realizaram 14 workshops commetodologia participativa e 23 entrevistas a profundidade que deramrazão de seudesenhoqualitativo e enfoque hermenêutico. Participaram 119 jovens registrados em programas das Faculdades de CiênciasSociais e Educação de universidades públicas e particulares (4 campus e 6 sedes), localizadas no departamento de Antioquia (Colômbia). Se identificou que os universitáriossão conscientes da presença do poder nasrelaçõescomseus pares e incluso com a instituição, mantémumavisão negativa do poder, lheassociamcom o abuso e o autoritarismo, normalmente veem como umator externo e muitopoucasvezes se reconhecem como quem os detêm e como caso atípico emergemalgumaspráticas de resistênciaemuma das universidades públicas, onde se associa o poder com o uso da violência como a única maneira, até o momento, que eles encontram para reverter o instituído.

Palavras chave: Educaçãouniversitária, poder, perspectivas e jovens universitários 


\section{Introducción}

Para las ciencias sociales ha sido una de sus prioridades el estudio de las relaciones que se generan entre seres humanos, siendo uno de sus mayores aportes la teorización de sus componentes, dinámicas y particularidades (Herrera, 2000). Ya diferentes disciplinas se han encargado de formular supuestos acerca de aquello que motiva al ser humano a relacionarse con otros y las tensiones que se forjan en ese contacto (Ibáñez y Mudarra, 2005, p. 106-107;Pfefferkorn, 2007).

Esas relaciones sociales se manifiestan en diferentes contextos que le dan lugar, por ejemplo, en las instituciones (escuela, familia, religión o empresas), los espacios públicos (calle, el bar y gimnasio), tecnologías de la información y la comunicación (redes sociales, blogs o correo electrónico), entre otros. La universidad es, entonces, un espacio apropiado para que germinen esas relaciones entre sus integrantes.

Otra de las características a señalar que se manifiesta en toda relación social, es que siempre hay intercambios entre sus actoresy constantemente se generan tensiones relacionadas con los usos y los abusos del poder. De hecho, el poder es uno de esos elementos que se manifiesta en el contacto social y que, sin ser la única fuerza que circula en él, es una realidad inseparable de su esencia.

Al intentar delimitar el término poder, según Múnera (2005), la múltiple concepción del vocablo en el lenguaje cotidiano hace que esta tarea sea compleja para las diferentes teorías; entonces esta cuestión hace que su definición pueda parecer ambigua. Por ejemplo, la palabra poder suele equipararse con dominación, violencia, potencia, autoridad, influencia o potestad; estas se mezclan en un mismo significado, que para este autor es impreciso y no apto.

Lo que sí es claro para Aguiló es que independientemente de su definición, “(...) El ejercicio del poder, en cualquiera de sus formas y manifestaciones, es un fenómeno prácticamente universal que puede detectarse en las relaciones sociales vigentes en las sociedades de todos los tiempos" (2009, p. 1). 
En esta investigación Michel Foucault fue de gran influencia para partir del poder como fuerza que simplemente es y que se manifiesta en todo tipo de relación. Por lo tanto, se hace necesario delimitar la categoría poder desde las propuestas de Foucault (1981), que según Sossa se pueden resumir en:

El poder se encuentra en el hombre mismo, no es algo externo que lo oprima o esclavice. En tal sentido, el razonamiento al poder, no consigue ser clasificado como algo bueno o malo, ya que el poder se esboza como una extensa red de relaciones (Sossa, 2011, p. 562).

Por su parte, Yela e Hidalgo (2010, p. 59) sintetizan la construcción que realizó Michel Foucault (2000) sobre el poder en la constitución de sujeto, al considerar que este autor se ha planteado el poder como ejercicio de orientar las posibles conductas y definir el campo de acción de los otros. Estos objetivos vinculados entre sí, garantizan la presencia del poder en las relaciones.

Por supuesto, debe entenderse que el poder no es una facultad que solo se asienta en unos cuantos, ya lo recuerda Ávila (2006),al citar las ideas de Michel Foucault (2000), cuando postula que "no es una propiedad, sino que es una estrategia. Es decir, el poder no se posee, se ejerce" (Ávila, 2006, p. 225).

Para Foucault (1978, citado en Rangel, 2009), el poder no se sabe bien quien lo tiene exactamente, pero parece que sí se tiene conocimiento de quien no lo ejerce, ya que siempre se ejerce con unos de una parte y los otros de la otra. De hecho, esta fue una característica identificada regularmente en el relato de los universitarios que participaron de esta investigación, ya que les fue más fácil ubicarse en el lugar de quien no lo detenta, que en aquellos sujetos que hacen uso de su poder.

García (2009), citando a Foucault (1992), hace énfasis al intentar definir el fenómeno del poder, partiendo de una visión tradicional, al comprender éste como el ejercicio de reprimir; pero esta delimitación hace del poder algo frágil, ya que le despoja de su condición constructiva. 
Hegel (2004, citado en García, 2009), devela la dinámica que se produce en una de las manifestaciones de poder, es decir que, con la dialéctica del amo y el esclavo, se evidencia que el poder no solo es una batalla por el predominio a través de la represión, sino que en esa condición que se impone de dependencia también hay una posición en aquel que se percibe como despojado de poder, una postura que facilita esa relación en el contacto con el otro; y que en los participantes de la investigación parece estar no consciente, ya que se perciben como abusados y no como abusadores, haciéndose evidente en expresiones como:"de mi han abusado más”.

Diferentes pensadores e intelectuales han hecho el ejercicio de delimitar su definición sobre aquello que se nombra como poder; por ejemplo, Max Weber (2002, citado en Múnera, 2005), le concibe como la "probabilidad de imponer la propia voluntad, dentro de una relación social” (p. 34); Henry Minztberg (1992, citado en Cruz, 2013), define el poder como "la capacidad de afectar (causar efecto en)..." (p. 47).

Por su parte Benbenaste, Delfino y Vitale (2006) proponen que desde la psicología el poder puede entenderse como "un tipo de vínculo, psíquicamente hablando, muy económico; que demanda escaso esfuerzo" (p, 356); y Arendt (1970, citada en Múnera, 2005), le concibe como "la capacidad humana de actuar..." (p, 36).

El poder es entonces una de esas dinámicas que conforman y constituyen las relaciones sociales; ya que éste surge en la manera de relacionarse entre las personas, grupos o instituciones. Este no debe entenderse de forma negativa, ya que perdería su carácter propositivo y creativo; no puede ubicarse en un solo actor que lo detenta y alguien que le carece, sino que circula constantemente entre los implicados en la relación. Tejiendo las concepciones que se tienen del poder, se puede definir como la capacidad para influir o causar efecto en otros, a través del vínculo que se establece.

Los hallazgos en la revisión teórica dan paso al interrogante sobre ¿cómo circula el poder en esas relaciones sociales que establecen los 
jóvenes dentro y fuera del aula de clase?. Surge entonces un interés por conocer sus experiencias y las construcciones que hacen como sujetos garantes de poder o, por el contrario, identificar si se perciben como despojados de éste.

Por lo tanto, se pone como centro y faro orientador de esta investigación una concepción imparcial del poder, con el fin de escuchar atentamente esas subjetividades de los participantes sin teñirles de ideas preconcebidas o de las visiones que aporta la teoría.

\section{Diseño metodológico}

Para orientar esta investigación y dar cumplimiento a su objetivo de comprender las experiencias subjetivas de universitarios con respecto a sus relaciones interpersonales, en este caso orientadas a la categoría del poder, se hizo uso del diseño cualitativo y el método hermenéutico.

Para Quecedo y Castaño (2002, p. 7) de manera general, puede definirse un enfoque cualitativo como la exploración que genera como resultado unos datos descriptivos, que pueden ser las propias palabras de las personas, verbales o escritas, y la conducta observable. Y según Gadamer (1977, citado en Valdivieso y Peña, 2007), en el "Círculo Hermenéutico”, la hermenéutica aporta al proceso de conocimiento, a través de la comprensión de la relación y no del objeto, ya que de manera holística se observa al fenómeno y la construcción de significados y no a sus partes.

En el trabajo de campo participaron 119 jóvenes (hombres y mujeres) matriculados en diferentes pregrados de Ciencias Sociales y Educación de diferentes Universidades, a través de 14 talleres donde se aplicaron técnicas interactivas como: construcción de relatos, foto-lenguaje, completación de frases, socio-drama y análisis de casos.

Los participantes pertenecían a las siguientes instituciones de educación superior del departamento de Antioquia (Colombia). Las de carácter privado fueron: la Corporación Universitaria Lasallista y la Universidad Cooperativa de Colombia; de perfil público: el Politécnico 
Colombiano Jaime Isaza Cadavid y la Universidad de Antioquia, Sedes Medellín, Carmen de Viboral y Santa Fe de Antioquia.

Consecutivamente, se eligieron 23 jóvenes para las entrevistas a profundidad, que representaran manifestaciones de la pluralidad: sexual, ideológica, religiosa, territorial y política.

Para dar cumplimiento en esta investigación a los lineamientos éticos que delimitan el quehacer del profesional en psicología, contenidos en la Ley 1090 de 2006 (Congreso de la República de Colombia, 2006), se dio cumplimiento a la aplicación de los principios de autonomía, justicia, beneficencia (Mondragón, 2007), confidencialidad, consentimiento informado, veracidad y fidelidad (Gonzáles, 2002; Ocampo, 2013); basados en la responsabilidad que tiene la ciencia con los sujetos que participan en investigaciones y el compromiso de los investigadores con la sociedad (Achío, 2003).

Se realizó un proceso de análisis de contenido que permitió organizar los datos construidos, hacer la tematización de los mismos, agruparlos por categorías (poder, conflicto y pluralidad) y consolidarlos en subcategorías.Se presentan a continuación por medio de una gráfica las dos subcategorías que se consolidaron con base a los resultados (ver tabla 1) y que se desarrollan en este artículo.

Tabla 1. Subcategorías de la categoría "Poder"

\section{PODER}

1.1 El poder como asimetría inevitable: perpetuando los abusos

1.2 Prácticas de resistencia como intentos de revertir lo instituido

Fuente: Autores

\section{Hallazgos}

El análisis de la información recolectada, con respecto a la categoría de poder, por medio de las técnicas aplicadas en los 14 talleres, dio paso a cuatro subcategorías que permiten comprender a mayor profundidad 
las experiencias subjetivas de los participantes. En este artículo se exponen dos (ver tabla 1) de esas subcategorías que arrojó la clasificación de los resultados.

La base teórica desde donde se abordó el concepto de “poder”, parte de la idea de concebirlo como una fuerza presente en todas las relaciones humanas (Penaglia, 2012; Foucault, 1976 y Foucault, 1981). De hecho, se identificó que los jóvenes son conscientes de su presencia en las relaciones que viven en el día a día en la universidad; aunque es necesario resaltar que suelen equipararle con una práctica negativa.

En este caso, para las categorías que se presentan en este artículo, los participantes suelen moverse en la dinámica de lo particular y lo colectivo, nosotros y ellos, aquí y allá; dado que se refieren a sus medios de organización para el uso y/o abuso del poder como grupo o individuo.

\section{El poder como asimetría inevitable: perpetuando los abusos}

Una tendencia en los relatos es que se reafirman dichos culturales tales como "el poder es pa' poder" y "el que manda, manda, aunque mande mal", pues como ya se ha mencionado, en general, el poder es visto como algo negativo, como una relación vertical, de imposición, abuso, opresión y unidireccionalidad. Que emplea mecanismos sutiles de manipulación y donde cada uno y cada una se considera el más afectado por dicha situación. Según una entrevista de un hombre de universidad pública:

"Cuando escuchamos la palabra poder nos permite una sensación negativa que normalmente la asociamos con abuso de poder"

Este abuso aparentemente prefijado y estático del poder, va en contravía de los planteamientos de Foucault (1996) cuando afirma que:

El poder tiene que ser analizado como algo que circula, o más bien, como algo que no funciona sino en cadena. No está nunca localizado aquí o allí, no está nunca en las manos de algunos, no es un atributo como la riqueza o un bien... En otros términos, el poder transita transversalmente, no está quieto en los individuos (p.145). 
Esto implica entonces, hacer otra lectura del poder como un fenómeno dinámico y en espiral, que supone tensión y potencia, que incluye a todos los individuos y por supuesto, está presente en todas sus relaciones.

\section{La manipulación es tan sutil...}

Pareciera que hay una suerte de desesperanza en relación con la posibilidad de transformar la disparidad en las relaciones de poder, como si ciertos hilos delgados y malintencionados tejieran una telaraña donde se puede caer atrapado, algo así como:

"Nos venden ese discurso y esa idea de que nosotros podemos cambiar asuntos, pero finalmente el que tiene el poder no lo cede, sea en una universidad pública o en una privada, porque eso trasciende el asunto académico e institucional; y eso es un asunto de estructura social desde su nacimiento" (Fragmento del debate del taller 2 donde se implementó la técnica de sociodrama).

Las expresiones de los jóvenes sobre el poder están relacionadas con verlo como la búsqueda de control y aprobación que orienta las acciones para influir en el otro, de modo que actúe como se desea, para obtener un beneficio más particular que general. Lo cual asocian a ganar, dominar, convencer, tener la razón, no ceder, aprovecharse, mentir, abusar, entre otras.

De otro lado, esta idea de lo sutil puede leerse desde la premisa de que las relaciones de poder, son tan difusas y dinámicas que no siempre alcanzan a ser institucionalizadas y que circulan en espacios sociales cotidianos, donde el sentido común adquiere mucha importancia, de tal forma que según Foucault (1996) “(...) El poder y sus ejercicios son dinámicos y en ocasiones sutiles; no es algo que se detente y que sea abrumadoramente avasallador. Tampoco es lineal o vertical” (p.144).

Por lo tanto, los universitarios cuando hablan de estrategias de poder hacen énfasis en cómo estas pueden ser usadas de forma directa o indirecta; pero la forma sutil es la que más les llama la atención, puesto que esta se refiere a la manipulación del otro.. Nuevamente esta idea se relaciona con la manera particular de percibir al poder como asimetría. 


\section{"De mí han abusado más..."}

Como ya se ha dicho, según los relatos de los participantes: los profesores tienen el poder porque evalúan y los directivos porque toman las decisiones administrativas, las cuales generalmente son percibidas como "abusos de poder" que sólo se dan en su universidad. Lo particular es que quienes estudian en universidad privada creen que esto no ocurre en la universidad pública y viceversa, tal como lo afirman estos jóvenes:

“...nosotros acá le atribuimos el poder a la institución porque le pagamos... y el capitalismo es así, quien tiene el dinero tiene el poder... mientras para una institución pública ellos no tienen el dinero, ellos son administrados por el Estado y cuando decimos Estado, sí le podemos reclamar como estudiantes..." (Fragmentos del debate del taller 1 en universidad privada donde se implementó la técnica de fotolenguaje).

“...hay cosas muy arbitrarias que se manejan, me imagino que es por ser una universidad privada y más católica, ¿́cierto?” (Fragmento de entrevista a hombre, de universidad privada).

Paradójicamente, desde la universidad pública se siente el mismo "abuso" institucional y se tiende a idealizar lo que pasa en la universidad privada, en palabras de una joven estudiante de universidad pública: "El poder en la universidad está mal encaminado de acuerdo con los intereses del pueblo" (Fragmento técnica de completación de frases).

Llama la atención que los estudiantes de las sedes de regionalización se consideran la "parte privada de la universidad pública" y, por tanto, creen que son menos escuchados y atendidos, tal como se observa a continuación:

“...no creo que implicaría un poder entre estudiantes, al menos en esta universidad. Tal vez en universidades donde el conflicto, digamos, es trasladado a la misma universidad, sea más evidente, digamos, como la Universidad Nacional o la Universidad de Antioquia en Medellín..." (Fragmento de entrevista a hombre, estudiante de universidad pública, sede regionalización). 
El asunto del "abuso del poder" también se evidencia en algunos relatos de manera diferenciada por género, es decir, mujeres que piensan que los hombres por su machismo no aceptan el poder que ellas ahora están "ganando"; y hombres que consideran que ellas tienen más privilegios especialmente si son "bonitas".

Esta idea del poder asociado a la verticalidad (en el género, en la relación académica, etc.), donde una persona gana y se impone y la otra pierde y se doblega, se ilustra mejor con el siguiente fragmento: "Lo que yo digo es que el poder es necesario, ¿para qué?, o sea tiene que haber una persona sumisa y una que domine, digámoslo así." (Entrevista a un hombre, estudiante de universidad pública)

Otro elemento que aparece relacionado con el poder es el "interés", es decir, un provecho que quieren obtener todas personas involucradas en una relación, una ventaja que desean sacar independiente de si el poder es de tipo físico, intelectual, económico, político, psicológico, etc.

Como caso atípico en los relatos de los jóvenes, se halla que el poder sea visto como favorable para regular la vida en común y que incluso sirve para ayudar a otros.

Entonces, como diría Foucault (1972): "Es preciso dejar de describir siempre los efectos del poder en términos negativos: 'excluye', 'reprime', 'rehúsa', 'abstrae', 'encubre', 'oculta', 'censura'. En efecto, el poder produce, produce lo real, produce campos de objetos y rituales de verdad..." (p.75).

Se puede concluir que independientemente de la condición particular de los jóvenes universitarios, es decir, que se estudie en una universidad pública o privada, que se sea afrodescendiente o mestizo, se defina como heterosexual u homosexual... se tiende a pensar que la propia condición siempre está en desventaja frente a la del otro.

A continuación, se va a exponer una subcategoría que, aunque parezca un poco más sutil y focalizada, solamente se presentó en algunos jóvenes de universidad pública, que se convierte en otra posibilidad del poder asociadas a las prácticas en la universidad. 


\section{Prácticas de resistencia como intentos de revertir lo instituido}

Como un caso particular, emergieron pocos relatos que hacen referencia a prácticas de resistencia, en muchas ocasiones asociadas a la manifestación violenta. Prácticas que no pretenden perpetuar el statu quo, sino transformar las acciones que consideran injustas y gestar cambios, aunque, según la percepción de quienes hacen referencia a este aspecto, sólo se logran a través de actos que "pongan a tambalear lo establecido", procesos de movilización que desemboquen en una "parálisis social" que realmente sea escuchada, pues según sus ideas, estos cambios no se logran sólo con lo que podría denominarse participación democrática. Esto se ilustra mejor con un fragmento de entrevista de un joven estudiante de universidad pública:

“...que cuando hay marchas de lo que sea, se ve la cantidad que hay y por qué están en contra de algo, toda esa gente, y ese día, la Gobernación y allá en la Alpujarra tiembla, tiembla la gente, sale en los periódicos, en todas partes, hasta el presidente se da cuenta, y es en ese momento, en donde ellos quieren que las cosas no pasen más allá...” (Fragmento de entrevista a un hombre, estudiante de universidad pública).

$\mathrm{Al}$ identificar sus prácticas para enfrentar las injusticias impartidas por otros como manifestaciones basadas en la violencia, se hallan posiciones divididas, hay quienes las apoyan, como en el testimonio de la joven que se presenta a continuación:

“... como el apoyo que se les puede dar a los muchachos sabiendo que están oponiendo la resistencia y que de pronto están cumpliendo un objetivo o una función de que se tiene el poder, de que la universidad todavía tiene como esa fuerza, que entonces no es opresible" (fragmento de una de las plenarias en universidad pública)

Hay quienes no están de acuerdo con las manifestaciones como práctica de resistencia, pues consideran nefastas sus consecuencias y, además, juzgan que estos actos también son otros modos de imposición, ya no por parte de las figuras instituidas, pero que igualmente usan la fuerza y la verticalidad. Los efectos negativos vulneran sus derechos tales como el de estudiar y ver su semestre académico en el pe- 
ríodo regular. Este asunto emerge en la voz de algunos participantes de un taller en universidad pública, así: “...la facultad que no quiso salir, no quiso como colaborar con la causa, entonces fueron hasta allá después, se dieron cuenta y fueron con una papa bomba" (fragmento de uno de los talleres realizados en una universidad pública).

Además, consideran que no siempre son actos argumentados. Este asunto emerge en la voz de algunos participantes de los talleres en las universidades públicas, así:

“...nosotros vemos eso como poder, porque uno va sintiendo que aquí en salón no se puede quedar, uno va saliendo obligadamente. Por ejemplo, lo que decía la compañera ahorita, sobre la facultad que no quiso salir, no quiso como colaborar con la causa, entonces fueron hasta allá después, se dieron cuenta y fueron con una papa bomba" (fragmento de una de las plenarias en universidad pública).

"yo ya este semestre para que voy a ir, si allá, sí sé que siempre es lo mismo: que sí votemos, votemos a ver si hay paro, entonces es lo que yo decía, por ejemplo, ahí también se ve evidenciado lo del poder. Votemos a ver si hay paro y votan y repiten la votación 4, 5, 6 [veces] y las que tengan que votar, hasta que digamos: sí vamos a paro, hasta que así sean los 4 estudiantes que estén en la asamblea, hasta que la gente se canse de venir" (fragmento de una de las plenarias en universidad pública)

En otras palabras, como diría Aguilera (2010) "La movilización y protesta juvenil oscila pendularmente entre adscripciones identitarias y conflicto social, y en su repertorio entre formas lúdicas y violentas, entre formas clásicas y otras emergentes, modificando el repertorio de movilización y acciones de contestación juvenil” (p. 87).

En síntesis, para esta subcategoría, cabe resaltar que solo unos pocos consideran que en su relación con lo que perciben como poder, brotan algunas prácticas de resistencia como intentos de revertir lo que desde otros se instituye como la única opción, y así mismo, dinamizar acciones de movilización que logren paralizar a quienes, desde el imaginario de los jóvenes, son abusadores del poder. 
A continuación se señalarán aquellos asuntos que desde el análisis de los resultados se identifican como contenidos ausentes en la subjetividad de los participantes o urgentes de ser profundizados en estudios posteriores al que se presenta.

\section{Discusión}

La universidad por ser un espacio de socialización (Arias y Alvarado, 2015), en donde los jóvenes interactúan constantemente y construyen relaciones entre ellos, se convierte en un lugar para que el poder se manifieste y tramite de diversas maneras. Los resultados de esta investigación dan cuenta de ello, en las dos subcategorías que se han expuesto, donde los participantes expresan su subjetividad en lo referente al poder, y la forma cómo circula en el contexto universitario.

En el análisis de los datos se identifica como caso atípico, presente en una de las universidades públicas, el que los participantes equiparan el uso del poder con sus experiencias de manifestación colectiva, es decir, en la forma de organización entre jóvenes con el fin de hacer resistencia violenta frente a quienes, según ellos, hacen uso del poder para imponerles un orden social predefinido. Se sugiere que esta situación sea abordada en próximas investigaciones.

Este asunto también genera la pregunta por las prácticas de resistencia no violenta, puesto que es una cuestión que se presenta en el discurso de algunos de los participantes de universidades públicas, pero que en estos tiende a tomar más la dimensión de los actos violentos, considerados por ellos como la única forma de transformar lo instituido.

Por lo tanto, al analizar sus prácticas surge el interrogante de si en los jóvenes universitarios cabe la posibilidad para pensar en métodos de resistencia sin violencia (Valenzuela, 2001), que de igual forma se perciba como una manifestación de podery no como su ausencia.

La cuestión de las diferencias entre la universidad pública y la privada, fue una situación que tomó mucha fuerza entre los participantes, ya que se manifestaban las inconformidades con la propia institución y se 
idealizaba la que tiene otro carácter en su estructura y funcionalidad. $\mathrm{Al}$ parecer, la tendencia a idealizar lo del otro en detrimento de lo propio, también se extiende a las experiencias de los jóvenes universitarios en relación con su pertenencia a una institución y no a otra.

Se sugiere profundizar en estas diferencias, e incluso semejanzas, con respecto al uso del poder en las instituciones privadas y públicas; para mostrar los movimientos que hay entre los usos y abusos del poder en alguna de ellas o si, por el contrario, circula en ambas al estar presente tanto el carácter de abuso como de altruismo.

Además, es necesario analizar el poder entre jóvenes universitarios no solo en los espacios formales tales como el aula de clase y los fenómenos que allí están en juego (el aprendizaje, el trabajo en equipo, la calificación, etc.), sino también en otros escenarios informales, relacionados con procesos aparentemente más simples y menos intencionados, concernientes a la socialización en la cafetería, las fiestas, las redes sociales, etc.

Uno de estos escenarios son las tecnologías de la información y la comunicación (TIC) (Cornejo y Tapia, 2011); es necesario entonces, ahondar en cómo se manifiesta el poder a través del WhatsApp, las publicaciones en Facebook, el que tiene más seguidores, los "me gusta" y los comentarios en las publicaciones, o en algo mucho más tajante como el aceptar o rechazar a alguien de contacto en estos medios.

Finalmente, es importante llamar la atención acerca de la necesidad de transformar esa concepción negativa que se tiene del poder (tanto propio como del otro), ya que, si se desvirtúan sus atributos, entonces no se hará un uso adecuado de este componente de las relaciones sociales y se perderá su energía que impulsa al desarrollo de las sociedades.

\section{Conclusiones}

Los jóvenes universitarios son conscientes de la presencia del poder en sus formas de relación, identifican algunos sujetos que según ellos son quienes lo detentan, en algunas vivencias se ubican a ellos mismos como garantes de poder y comprenden la organización entre ellos como una de las tantas posibilidades de ejercerlo. 
En el plano subjetividad el poder tiende a polarizarse en la dimensión negativa, aunque surgió el caso atípico de reconocer su cualidad creativa, propositiva, colaborativa; pero sí es una particularidad el entenderlo como una fuerza cuya finalidad es la represión, el abuso, el dominio, la violencia e imposición, e incluso la organización entre los universitarios para revertir lo instituido se canaliza desde la violencia como la única forma de lograr su objetivo.

Los asuntos que requieren de atención en próximas investigaciones y que surgen a partir del proceso de análisis se pueden agrupar en: las prácticas de resistencia no violenta; el uso del poder a través de las tecnologías de la información y la comunicación; y las diferencias en el uso del poder en la universidad pública y privada.

El poder, por lo tanto, se manifiesta en todas las relaciones sociales, no es un bien que lo poseen unos y otros no, sino que circula en los sujetos a través de las relaciones que establecen, convirtiéndose en una característica que no se puede desligar de ninguna relación humana. 


\section{Referencias}

Achío, M. (2003). Los comités de ética y la investigación en Ciencias Sociales. Revista de Ciencias Sociales 1(99), 85-95. Recuperado de:http://www.redalyc.org/ pdf/153/15309907.pdf

Aguilera, O. (2010). Acción colectiva juvenil: De movidas y finalidades de adscripción. Nómadas, (32), pp. 81-98. Recuperado de: http://www.redalyc.org/ pdf/1051/105114733006.pdf

Aguiló, A. (2009). El concepto de «poder» en la teoría política contrahegemónica de Boaventura De Sousa Santos: una aproximación analítico-crítica. Nómadas, 24, 1-21. Recuperado de:http://www.iberopuebla.mx/microSitios/catedraTouraine/articulos/EL\%20CONCEPTO\%20DE\%20\%C2\%ABPODER\%C2\%BB\%20EN\%20LA\%20TEOR\%C3\%8DA\%20POL\%C3\%8DTICA\%20CONTRAHEGEM\%C3\%93NICA\%20DE.pdf

Arias, A., y Alvarado, S. (2015a). Jóvenes y política: de la participación formal a la movilización informal. Revista Latinoamericana de Ciencias Sociales, Niñez y Juventud, 13(2), 581-594. Recuperado de: http://revistalatinoamericanaumanizales.cinde.org.co/wp-content/uploads/2015/o8/J\%C3\%B3venes-y-pol\%C3\%ADtica vol13n2a2.pdf

Ávila, F. (2006). El concepto de poder en Michel Foucault. Telos, 8(2), 215-234. Recuperado de http://www.redalyc.org/articulo.oa?id=99318557005

Benbenaste, N., Delfino, G.\&Vitale, N. (2006). La contribución de la psicología al concepto de poder. Psycholy, 5(2), 351-360. Recuperado de: http://pepsic. bvsalud.org/pdf/up/v5n2/v5n2a11.pdf

Cornejo, M., y Tapia, M. (2011). Redes sociales y relaciones interpersonales en internet. Fundamentos en Humanidades, 12(24), 219-229. Recuperado de: http:// www.redalyc.org/pdf/184/18426920010.pdf

Cruz, L. (2013). El concepto de poder en la administración. Contaduría y Administración 58(4), 39-57. Recuperado de: http://www.scielo.org.mx/pdf/cya/ 
v58n4/v58n4a3.pdf

Foucault, M. (1972). Historia de la sexualidad. México: Siglo XXI.

Foucault, M. (1976). Vigilar y castigar. México: Siglo XXI, México.

Foucault, M. (1981). Un diálogo sobre el poder. Barcelona: Alianza.

Foucault, M. (1996). Microfísica del poder. Madrid: Editorial de la Piqueta.

García, Ó. (2009). El concepto de poder y su interpretación desde la perspectiva del poder en las organizaciones. Estudios Gerenciales, 25(110), 63-83. Recuperado de: http://www.redalyc.org/articulo.oa?id=21219323004

Gonzáles, M. (2002). Aspectos éticos de la investigación cualitativa. Revista Iberoamericana de Educación, 29, 85-103. Recuperado de: http://www.redalyc. org/pdf/800/80002905.pdf

Herrera, M. (2000). La relación social como categoría de las ciencias sociales. Revista Española de Investigaciones Sociológicas, 90, 37-77. Recuperado de: http:// www.redalyc.org/pdf/997/99717877002.pdf

Ibáñez, P., y Mudarra, M. (2005). Relaciones sociales en el empleo, en trabajadores con discapacidad. Educación XX1, 8, 105-125. Recuperado de: http://www. redalyc.org/pdf/706/70600805.pdf

Mondragón, L. (2007). Ética de la investigación psicosocial. Salud mental, 3o(6), 2531. Recuperado de: http://www.redalyc.org/pdf/582/58230604.pdf Múnera,L. (2005). Poder (Trayectorias teóricas de un concepto). Colombia Internacional, 62, 32-49. Recuperado de: http://www.redalyc.org/ pdf/812/81206203.pdf

Ocampo, J. (2013). Bioética y psicología clínica: reflexiones. Anales médicos, 58(4), 253-259. Recuperación de: http://www.medigraphic.com/pdfs/abc/bc-2013/ bc134f.pdf

Penaglia, F. (2012). La paradoja del poder: ¿Elitización o empoderamiento colectivo? Polis, 11(32), 105-118. Recuperado de: http://www.scielo.cl/pdf/polis/v11n32/ art07.pdf 
Pfefferkorn, R. (2007). El trabajo, un eje fundamental de las relaciones sociales de sexo. Revista de Ciencias Sociales (Cl), 18, 53-70. Recuperado de: http:// www.redalyc.org/pdf/708/70801804.pdf

Quecedo, R., \& Castaño, C. (2002). Introducción a la metodología de investigación cualitativa. Revista de Psicodidáctica, 14, 5-39. Recuperado de: http://www. redalyc.org/pdf/175/17501402.pdf

Rangel, P. (2009). La vigencia del concepto de poder de Michel Foucault. Compendium, 12(23), 49-66. Recuperado de: http://www.redalyc.org/ pdf/880/88015905004.pdf

Congreso de la República de Colombia. (2006). Ley 1090 Por la cual se reglamenta el ejercicio de la profesión de Psicología, se dicta el Código Deontológico y Bioético y otras disposiciones. Bogotá: Congreso de la República.

Sossa, A. (2011). Análisis desde Michel Foucault referentes al cuerpo, la belleza física y el consumo. Polis, 1O(28), 559-581. Recuperado de: http://www.scielo.cl/ pdf/polis/v10n28/art26.pdf

Valdivieso, F., \& Peña, L. (2007). Los enfoques metodológicos cualitativos en las ciencias sociales: una alternativa para investigar en educación física. Laurus, 13(23), 381-412. Recuperado de: http://www.redalyc.org/pdf/761/76102319.pdf

Valenzuela, P. (2001). La no violencia como método de lucha. Reflexión Política, 3(5), 1-11. Recuperado de: http://www.redalyc.org/pdf/110/11000506.pdf

Yela, J., \& Hidalgo, C. (2010). El poder en Foucault: bases analíticas para el estudio de las organizaciones. Cuadernos de Administración, 44, 57-70. Recuperado de: http://www.redalyc.org/pdf/2250/225017586004.pdf 